\title{
Retinopathy of prematurity: preferred practice patterns among pediatric ophthalmologists
}

This article was published in the following Dove Press journal: Clinical Ophthalmology

\author{
Samiksha Fouzdar Jain' \\ Helen H Song ${ }^{2}$ \\ Shaza N Al-Holou ${ }^{2}$ \\ Linda A Morgan' \\ Donny W Suh ${ }^{1,2}$ \\ 'Ophthalmology Department, \\ Children's Hospital and Medical \\ Center, Omaha, NE, USA; ${ }^{2}$ Truhlsen \\ Eye Institute, University of Nebraska \\ Medical Center, Omaha, NE, USA
}

Correspondence: Linda A Morgan Ophthalmology Department, Children's Hospital and Medical Center, 8400

Dodge St, Omaha, Nebraska 68I I4, USA

Tel +l 40249058 I5

Email limorgan@childrensomaha.org

\begin{abstract}
Purpose: The treatment of retinopathy of prematurity (ROP) is not standardized and can vary significantly between providers. This study aims to determine preferred practices in treating ROP by globally surveying pediatric ophthalmologists.

Methods: Between January and February 2017, an international pediatric ophthalmology interest group was invited to complete an anonymous survey of 18 questions. The main objectives were to determine the preferred first line of treatment for ROP, the preferred dosage of intravitreal bevacizumab (IVB) used, and the outcome and possible complications following bevacizumab injection.

Results: Out of 101 pediatric ophthalmologists, 72 (71.8\%) stated that they had direct involvement in the treatment of ROP. When presented with type 1 ROP which requires treatment, 69 ophthalmologists $(68.3 \%)$ stated that they prefer laser treatment over bevacizumab, and 33 ophthalmologists $(32.7 \%)$ stated they would recommend bevacizumab as a first choice. Ninety-three ophthalmologists (92.1\%) reported the success of 1 laser treatment between $75 \%$ and $100 \%$, and 35 ophthalmologists (34.7\%) perceive bevacizumab to be $75 \%-100 \%$ successful. Half dose of adult-prescribed bevacizumab at $0.625 \mathrm{mg} / 0.05 \mathrm{~mL}$ was preferred by 47 of the ophthalmologists (46.5\%). No cases of endophthalmitis were reported with intravitreal injection. Conclusion: Laser photoablation remains the preferred mode of treatment for ROP among surveyed ophthalmologists across the world. Though bevacizumab is currently being used, this form of treatment is not as common, primarily due to the unknown safety profile and potential long-term ramifications of the drug.
\end{abstract}

Keywords: retinopathy of prematurity, intravitreal bevacizumab, neonatal intensive care unit, laser photoablation

\section{Introduction}

The treatment of retinopathy of prematurity (ROP) is not currently at an agreement. The departure from cryotherapy in the 1990s has segued into the current universally accepted modality of laser photoablation. Most pediatric ophthalmologists subjectively view laser as an effective treatment; however, the laser is not absolved of significant potential side effects. ${ }^{1}$ The ETROP study has established the guidelines currently in place for treating type I and severe ROP with conventional laser therapy. The study shows that conventional laser therapy for zone I ROP is successful in about half the cases, but when multiple attempts of laser fail to induce regression, a vitrectomy is required. ${ }^{2}$ Several case studies involving anti-vascular endothelial growth factor (anti-VEGF) treatment such as intravitreal bevacizumab (IVB) have reported using half of the adult dosage in pediatric patients with successful regression of ROP, including the BEAT-ROP study, which ascertained the significant efficacy of treating zone I ROP disease with bevacizumab. However, injecting an anti-VEGF modality into a 
preterm infant poses noteworthy risks, including endophthalmitis, pulmonary hypoplasia, and stroke, as well as tractional retinal detachments which may occur up to a year after initial injection. ${ }^{3}$ The mechanism of action of anti-VEGFs lends itself to potentially deleterious effects in the developing infant, and the lack of long-term follow-up data deters many who seek alternative ROP treatments.

As new methods of treatment are explored, optimal protocols to manage ROP have to be established. Bevacizumab injections are gaining popularity in treating ROP due to the ease of the procedure, preservation of peripheral vision fields, and effectiveness in reducing recurrence events after initial treatment. ${ }^{3}$ However, as the drug is currently being administered off-label of the US Food and Drug Administration recommendations, the method of management is not done in unanimity.

Despite numerous case studies and 1 multicenter, randomized control study, administration and management protocols for bevacizumab in preterm infants with ROP have not been established. This study aims to determine preferred practices in treating ROP by surveying pediatric ophthalmologists around the world. The objectives hope to address the preferred method of first-line treatment, the use of bevacizumab, the most commonly administered dosing of the medication, and to identify the outcomes and potential complications during follow-up examinations.

\section{Methods}

Approximately 1,000 members of the Pediatric Ophthalmology International ListServ (PED-OPHTH-L) were invited from January to February 2017 to complete an anonymous questionnaire via surveymonkey (http:/www.surveymonkey.

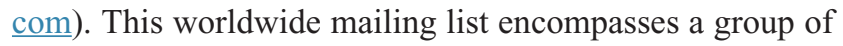
pediatric ophthalmology and strabismus physicians and is maintained at the University of California, San Diego, CA, USA. Per the University of Nebraska Medical Center institutional review board, informed consent was implied upon responders' completion of the survey. The responders did not include their location of practice. All procedures and data collection were conducted in a manner compliant with the Health Insurance Portability and Accountability Act of 1996.

The survey was composed of 18 questions and was distributed a total of 3 times in order to get the maximum number of responses (Box 1). Data in this study was included from the participants who answered yes or no to "Do you yourself treat the ROP patient (with laser or injection) following your diagnosis?" Therefore, results are presented for the participants who are actively involved in the diagnosis
Box I Survey questionnaire

I) Do you yourself treat the ROP patient (with laser or injection) following your diagnosis?

a. Yes

b. No

2) Who performs the ROP treatments (laser or injection) at your institution?

a. Yourself

b. Retina specialists

c. Refer/transfer care to different institution

d. Other

3) Do you use Ocular Imaging System (ie, RetCam) to obtain images to assist with the management of ROP?

a. Yes

b. No

4) With what therapy would you begin treatment for ROP with type I disease?

a. Confluent laser

b. Avastin injection (skip to question 8 if selecting this choice)

c. Other anti-VEGF medication

d. Cryotherapy

e. No treatment and observe

f. Other

5) If using laser as first-line therapy, what is your rationale for using this modality initially?

a. Personal preference

b. Avastin is not available

c. Avastin is not supported by my institution

d. Unknown safety profile of Avastin

e. Avastin not FDA approved

f. Other

g. N/A

6) If you were to use laser, what location would you perform the laser treatment?

a. NICU

b. Operation room

c. Other

7) How successful do you perceive I laser treatment is for most type I patients?

a. $10 \%$ effective

b. $25 \%$ effective

c. $50 \%$ effective

d. $75 \%$ effective

e. $100 \%$ effective

f. Other

8) What is your follow-up protocol after first treatment with the laser?

a. Every week for 4 weeks

b. Every other week for 4 weeks

c. Other

9) If laser has failed after I session, what is your next plan of treatment?

a. Laser treatment

b. Avastin injection

c. Other anti-VEGF medication

d. Refer to another colleague

e. Other

10) If using Avastin for treatment (first line or second line), what dose would you use?

a. $1.25 \mathrm{mg} / 0.05 \mathrm{~mL}$

b. $0.625 \mathrm{mg} / 0.05 \mathrm{~mL}$

(Continued) 
Box I (Continued)
c. $0.375 \mathrm{mg} / 0.03 \mathrm{~mL}$

d. Other

II) How successful do you feel I injection of Avastin is for most type I patients?

a. $10 \%$ effective

b. $25 \%$ effective

c. $50 \%$ effective

d. $75 \%$ effective

e. $100 \%$ effective

f. Other

12) After initial Avastin injection, how soon do you follow-up with the patient to check for regression?

a. I day

b. 2 days

c. 3 days

d. 4 days

e. I week

f. Other

13) What is your standard time frame for monitoring patients after the initial follow-up visit?

a. Weekly for I-2 months

b. Weekly for 3-4 months

c. Other

14) If you note a failed initial treatment with a patient after Avastin, what mode of treatment would you consider next?

a. Second Avastin injection

b. Laser

c. Other

15) How long do you wait to see improvement in the ROP prior to next treatment?

a. 5 days

b. 7 days

c. 10 days

d. 14 days

e. Other

16) How many times would you be comfortable reinjecting Avastin after the initial injection?

a. I time

b. 2 times

c. 3 times

d. Other

17) Have you noted any complications that may or may not correlate to the developing infant after Avastin injection?

a. Endophthalmitis

b. Systemic hypertension

c. Strokes

d. Pulmonary underdevelopment

e. Other

18) What is your postop antibiotics regimen following Avastin injections?

a. Antibiotic eye drops for 3 days

b. Topical betadine immediately after procedure followed by antibiotic drops

c. Topical betadine immediately after procedure (with no antibiotic drops)

d. No postop antibiotic care

e. Other

Abbreviations: FDA, US Food and Drug Administration; N/A, not applicable; $\mathrm{NICU}$, neonatal intensive care unit; ROP, retinopathy of prematurity; VEGF, vascular endothelial growth factor. and management of ROP. Some data do not add up to $100 \%$ as respondents were allowed to have more than 1 response to several questions. The Pearson $\chi^{2}$ exact test was used to calculate $p$-values, and descriptive data will be presented. Ethical approval for this study was granted by the University of Nebraska Medical Center Institutional Review Board (IRB 034-17EX).

\section{Results}

Out of 1,000 members, 101 pediatric ophthalmologists responded to the survey, leading to a response rate of $10.1 \%$. Of the 101 who responded, $72(71.3 \%)$ were directly involved in treatment ROP, while 29 (28.7\%) did not treat ROP patients by either laser or injection following diagnosis. Of the 72 ophthalmologists who performed treatment, $71(98.6 \%)$ did the procedure themselves while $8(11.1 \%)$ relied on retina colleagues to perform the treatment.

For 59 (58.4\%) respondents, management of ROP was assisted solely through clinical examination compared to 42 (41.6\%) participants who were aided by an ocular imaging system, such as RetCam ${ }^{\circledR}$ (Natus Medical Incorporated, San Carlos, CA, USA). Of those 42 ophthalmologists, 1 utilized Optos $^{\circledR}$ widefield imaging (Optos, Inc., Marlborough, MA, USA) to assist in ROP diagnoses.

Sixty-nine ophthalmologists (68.3\%) would treat type I ROP patients with laser as the initial treatment modality, while 33 (32.7\%) would employ bevacizumab, with 20 of those ophthalmologists stating that their decision is dependent on the zone of ROP involvement. None of the ophthalmologists would perform cryotherapy for their patients.

Ophthalmologists who did not use bevacizumab in their treatment repertoire most commonly cited unknown safety profiles of the drug as to why they preferred laser treatment (Table 1). Lack of US Food and Drug Administration approval as well as established national guidelines may

Table I Physician rationale for using a laser as the first treatment versus injection of IVB

\begin{tabular}{ll}
\hline Reasons & $\mathbf{N}(\%)$ \\
\hline Personal preference & $22(30.5)$ \\
Avastin not available & $3(4.17)$ \\
Not supported by institution & $7(9.72)$ \\
Unknown safety profile of IVB & $39(54.16)$ \\
Not FDA approved & $3(4.17)$ \\
Location (zone) of the disease & $7(9.72)$ \\
Following national guidelines & $2(2.78)$ \\
Frequent follow-up required with Avastin injection & $2(2.78)$ \\
Parent refusal for Avastin & $\mathrm{I}(\mathrm{I} .38)$ \\
\hline
\end{tabular}

Note: Physicians were able to choose more than I answer.

Abbreviations: FDA, US Food and Drug Administation; IVB, intravitreal bevacizumab. 
contribute to the unknown versus accepted safety profile of bevacizumab. Personal preference was noted as the second most chosen answer, surpassing home institution support as well as availability of the drug.

The home institution's neonatal intensive care unit was the preferred site to perform laser treatment for 64 ophthalmologists (63.4\%), while 40 (39.6\%) would use an operating room for performing laser treatment. In some instances, the chosen treatment location varied according to facilities available at that particular site. Alternatively, some physicians routinely utilized neonatal intensive care unit for all of their inpatient procedures and the operating room for outpatients, while very rarely the availability of the anesthesia staff influenced the treatment location.

Over $60 \%$ of the ophthalmologists $(n=64)$ would examine the patients weekly for 4 weeks post laser treatment, and only $6(5.9 \%)$ would follow-up every other week for 4 weeks. From the free text comments, most ophthalmologists would like to see the patient until full regression of the disease, with follow-up protocols varying.

The physicians were asked how they perceive laser treatment and its effectiveness. Over $92 \%$ of the ophthalmologists perceive that 1 laser treatment was more than $75 \%$ successful in treatment. If laser treatment failed after 1 session, 67 (66.3\%) physicians would perform repeat laser while $28(27.7 \%)$ would consider IVB injection. Seven $(6.9 \%)$ would consider other anti-VGEF agents. Most of the ophthalmologists would fill in skip areas with laser first and would consider IVB if there were no skip areas.

Of those using bevacizumab, 38 (52.78\%) recommend dosage of bevacizumab injections at $0.625 \mathrm{mg} / 0.05 \mathrm{~mL}$, $7(9.72 \%)$ used $0.375 \mathrm{mg} / 0.03 \mathrm{~mL}$, and $3(4.17 \%)$ used $1.25 \mathrm{mg} / 0.05 \mathrm{~mL}$ (Table 2).

After initial IVB injection, follow-up protocols ranged from 1-7 days (Figure 1). Most ophthalmologists looked for injection-related complications including cataracts or infectious endophthalmitis during the immediate follow-up period.

Table 2 Dosage of bevacizumab used for treatment of ROP

\begin{tabular}{ll}
\hline Dosage & $\mathbf{N}(\%)$ \\
\hline $0.25 \mathrm{mg} / 0.01 \mathrm{~mL}$ & $3(4.16)$ \\
$0.3125 \mathrm{mg} / 0.0125 \mathrm{~mL}$ & $2(2.77)$ \\
$0.375 \mathrm{mg} / 0.03 \mathrm{~mL}$ & $7(9.72)$ \\
$0.5 \mathrm{mg} / 0.02 \mathrm{~mL}$ & $1(1.38)$ \\
$0.625 \mathrm{mg} / 0.025 \mathrm{~mL}$ & $4(5.55)$ \\
$0.625 \mathrm{mg} / 0.05 \mathrm{~mL}$ & $38(52.78)$ \\
$0.75 \mathrm{mg} / 0.03 \mathrm{~mL}$ & $2(2.77)$ \\
$1.25 \mathrm{mg} / 0.05 \mathrm{~mL}$ & $3(4.17)$ \\
\hline
\end{tabular}

Abbreviations: IVB, intravitreal bevacizumab; ROP, retinopathy of prematurity.

\section{Recommended follow-up after injection}

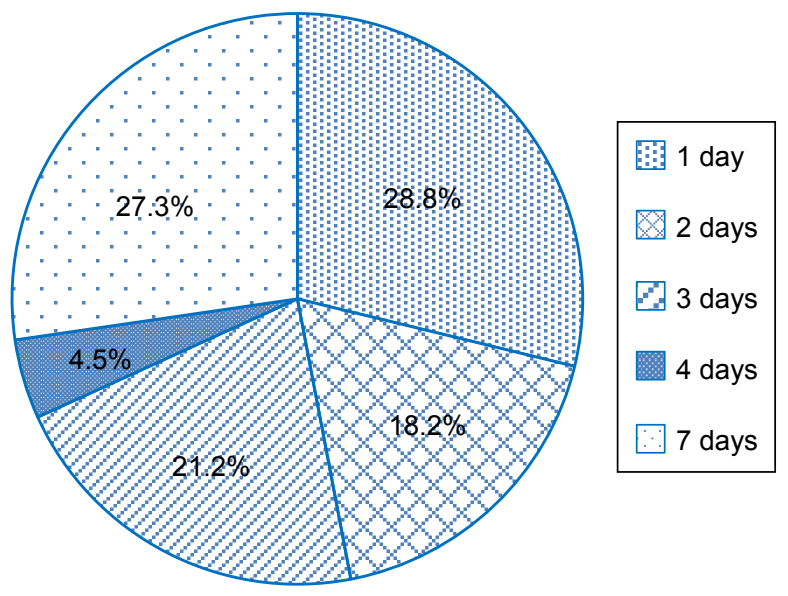

Figure I First follow-up appointment after injection.

In the survey, only about a third of the participants perceived IVB as being more than $75 \%$ successful in treatment for type 1 ROP. Many surveyed ophthalmologists stated that they were not comfortable responding to success rates due to low treatment rates in their clinics. Some have reported recurrence of the disease following IVB. Also, the patients are followed up longer until full vascularization of the retina or postmenstrual age of 70 weeks following an IVB injection compared to after laser treatment.

After failed bevacizumab injections, 51 ophthalmologists $(50.5 \%)$ would perform laser as a second procedure and $14(13.9 \%)$ would consider repeating anti-VEGF injection. The selection of the second procedure is based on many factors including the medical stability of the child, disease zoning and staging, and family preferences. Ophthalmologists stated that if the child was stable, they would perform laser during a course of bevacizumab injection. However if they were unstable, IVB would be the preferred method. In addition, some responses stated evaluating the disease process for a particular child was determinant for the second procedure. Patients with zone 1 ROP would receive bevacizumab while those with zone 2 and 3 would be treated by laser. Others would refer to a retina specialist.

Before performing a second anti-VEGF injection, 39 ophthalmologists (38.6\%) would wait for 1 week before injecting, while $9(8.9 \%)$ would perform an injection on day 5. Fifteen (14.9\%) would wait up to 14 days before reinjection. Following initial IVB, 45 (44.6\%) practitioners would perform repeat IVB injection only once, while $25(24.8 \%)$ would perform injection twice for a patient. None of the ophthalmologists would repeat IVB 3 times for their patients. 
Table 3 Complications following IVB injection

\begin{tabular}{ll}
\hline Complications & $\mathbf{N}(\%)$ \\
\hline None & $67(66.3)$ \\
Systemic hypertension & $0(0)$ \\
Stroke & $0(0)$ \\
Pulmonary underdevelopment & $\mathrm{I}(1.0)$ \\
Endophthalmitis & $0(0)$ \\
Vitreous hemorrhage & $\mathrm{I}(1.0)$ \\
Myopia & $\mathrm{I}(1.0)$ \\
Prolonged time for retinal maturity & $\mathrm{I}(1.0)$ \\
Developmental delay & $3(2.9)$ \\
Demise/death & $2(2.0)$ \\
Other, not specified & $27(26.7)$ \\
\hline
\end{tabular}

Abbreviation: IVB, intravitreal bevacizumab.

Of the survey respondents, 67 ophthalmologists $(66.3 \%)$ had not noted any ocular or systemic complications following IVB injection. However, a single ophthalmologist reported pulmonary hypoplasia. Others submitted ocular and systemic problems not listed in the survey, including vitreous hemorrhage (1), myopia (1), prolonged time for retinal maturity (1), developmental delay (3), and infant demise (2), (Table 3). None of them reported endophthalmitis following IVB injection. It is not clear from the comments if vitreous hemorrhage was secondary to IVB. Sixty of the ophthalmologists (59.4\%) used postop antibiotics at least for 3 days, and the duration could be longer based on the survey.

\section{Discussion}

This study sought to investigate the preferred practice patterns of pediatric ophthalmologists and strabismus surgeons for the management of ROP. The traditional preferred mode of treatment has long been laser photocoagulation. This is mirrored in the primary finding of this survey: over $68 \%$ of surveyed ophthalmologists preferred laser treatment over anti-VEGF injections as an initial treatment for ROP. Due in large part to the lack of consensus on bevacizumab's safety profile, physicians also preferred laser due to the laser's long-term safety record and evidence-based treatment, as well as bevacizumab follow-up logistics, risks, and lack of long-term studies. To date, there has been only 1 prospective, controlled, randomized, multicenter clinical trial and many retrospective or prospective studies since 2010 studying the efficacy of bevacizumab on ROP regression..$^{4-9}$ In the 1 prospective study, the BEAT-ROP clinical trial, retreatment in laser-treated patients occurred more frequently and with more peripheral retinal damage than in injection-treated patients, suggesting anti-VEGF therapy as a beneficial alternative treatment for zone I ROP patients. ${ }^{5}$
Caution, however, needs to be heeded with the use of anti-VEGF agents as VEGF itself plays a critical role in the development of fetuses and newborns, particularly in premature infants who may further require pulmonary and neurological development and whose retinal-blood barrier may not be fully established. ${ }^{10,11}$ In this survey, systemic complications of bevacizumab use included developmental delay and pulmonary under development cited by 4 ophthalmologists and infant demise by 2 ophthalmologists. The majority of participants surveyed, however, did not find any complications with bevacizumab use, and many previous studies have not found systemic side effects in patients treated with bevacizumab. ${ }^{4,8,9,12-14}$

As the risk of anti-VEGF therapy includes potential systemic side effects, numerous studies have emerged which evaluate these effects. In 1 retrospective neurodevelopment study on 2-year olds who received ROP treatment as infants, there was a statistically significant increase in severe psychomotor developmental delays in patients treated with IVB and laser versus those treated with laser alone. However, comparing laser treatment alone to IVB treatment alone, no statistically significant difference was found in neurodevelopment. ${ }^{15}$ Another study demonstrated decreased serum VEGF levels in 7 ROP patients following bevacizumab injection. Interestingly, no adverse side effects were noted in these patients. ${ }^{16}$ Despite the fact that the majority of practicing ophthalmologists have experienced no complications with bevacizumab, the few reported complications are severe, likely contributing to ophthalmologists' reticence to adopt anti-VEGF injection therapy as initial ROP treatment.

While the majority of pediatric ophthalmologists preferred to use the RetCam for diagnostic imaging of ROP, 1 responder named Optos as the preferred method. Upon further investigation, 1 study found that the dual confocal-like laser system of Optos allowed for a faster image acquisition time, and thus the erratic and uncontrolled ocular movements of infants was less of a hindrance. In addition, that same study noted that excessive pressure on the eye elicited by the RetCam inhibits accurate imaging of plus disease. ${ }^{17}$

For ophthalmologists who utilize both treatment methods, many responded that zone I or aggressive posterior zone II ROP patients would receive bevacizumab, whereas most zone II ROP patients would receive laser treatment. This follows previous studies, particularly the BEAT-ROP, demonstrating efficacy with bevacizumab for zone I disease. ${ }^{5,13}$ Bevacizumab is preferred in patients with hazy corneas, iris neovascularization, or small pupils, where laser treatment would be difficult. On the other hand, parental or 
neonatologist preference, long-standing evidence, national guidelines and recommendations, and evidence of relapse with bevacizumab were cited as reasons in support of performing laser over IVB. It should be noted that this was an anonymous, international survey where regulations varied by practice location.

Dosages of bevacizumab treatment and its safety profile continue to be debated. For physicians using bevacizumab treatment, about half recommend the $0.625 \mathrm{mg}$ dosage. This dosage, half the typical dosing for adults for treating choroidal neovascularization and diabetic macular edema, was recommended from the BEAT-ROP study. ${ }^{18}$ Given its efficacy in this major prospective clinical trial, it is likely felt to be a standard dose for pediatric ophthalmologists treating ROP. A lower dosage of $0.375 \mathrm{mg}$ has been suggested more recently, ${ }^{19}$ and a separate study found that a dosage of $0.031 \mathrm{mg}$ was effective treatment for ROP and could reduce systemic side effects. ${ }^{20}$ Other anti-VEGF agents are also being tested as alternative therapies. Bevacizumab itself is a relatively large molecule with a half-life of approximately 2 weeks. Ranibizumab, a smaller molecule with a half-life of about 3 days and therefore less potential for deleterious systemic side effects, is found to be effective in treating bilateral aggressive posterior ROP in dosages as low as $0.12 \mathrm{mg}$, less than a quarter of the standard adult dosage. ${ }^{21}$

Pediatric ophthalmologists surveyed differed on how frequently and how quickly they followed up with patients after bevacizumab injection. Given the lack of comparative trials determining appropriate and standardized follow-up, physicians treating ROP likely will follow-up based on their own comfort with the medication. It has been suggested that bevacizumab injection may require longer follow-up to ensure a favorable outcome. , $22^{2}$ One study of 20 bevacizumab-treated ROP eyes showed peripheral and posterior pole abnormalities including vessel leakage, shunts, and tangles in patients 4 years of age. ${ }^{23}$ Also, when evaluating fluorescein angiograms between 44 and 150 weeks of age of 30 ranibizumab-treated eyes, vascularization to zone III occurred in only $50 \%$ of eyes, and most of the 30 eyes had evidence of vascular abnormalities. ${ }^{24}$ Indeed, surveyed ophthalmologists note that longer follow-up is the cause for preferring laser treatment in order to ensure adequate ROP regression, especially in patients who may not be followed up adequately.

The majority of practitioners surveyed feel their preferred treatment method is effective the majority of the time. Previous studies, including the BEAT-ROP study, have found injection to be more efficacious than laser treatment in the zone I patients. ${ }^{15}$ While our finding is limited given that the answer is anecdotal and not quantitative, it does demonstrate confidence in pediatric ophthalmologists' treatment for ROP despite the variation in method.

Though 1 study has demonstrated the efficacy of the use of bevacizumab injection as an adjunct to laser, only 28 of our survey participants $(27.7 \%)$ would recommend bevacizumab for adjunctive treatment and only after failed initial laser treatment. ${ }^{12}$ Perhaps this is due to lack of availability of bevacizumab, or the need to first treat skip lesions by laser before converting to a different treatment method. In contrast, if patients failed bevacizumab treatment, surveyed pediatric ophthalmologists were more likely to switch to laser treatment. This has been shown to be an effective method to halt ROP progression, but it could stem from the worry that a second injection would decrease VEGF levels in the body, possibly increasing adverse events. ${ }^{6}$

Certainly, limitations exist in our study, including the survey's reliance on physician input. Of more than 1,000 interest group members who were invited to fill out the survey, only 101 responded, leading to a $10.1 \%$ response rate. This relatively small response rate is a weakness in the study as some results are generalized conclusions and not statistically significant. However, while 72 responders (71.3\%) said they managed ROP at their institution, it is likely that ophthalmologists who did not treat ROP did not participate in the study at all. Further, this survey did not poll general ophthalmologists or retina specialists who may be the primary providers treating and monitoring patients for ROP. Indeed, 29 pediatric ophthalmologists $(28.7 \%)$ stated that a retina specialist performed ROP evaluations and treatment at their institution. Despite these limitations, our study obtained more than 70 physicians who do provide ROP management, which we feel adequately captures ROP practice patterns of ophthalmologists.

In addition, due to the rigidity of the survey format, clarifications were not given for many answers. For example, it is not clear that whether vitreous hemorrhage was secondary to IVB injection or a part of the disease. However, most questions offered a text box in which those surveyed could extrapolate their answers, which many ophthalmologists did use. Myopia should also be considered as an early or late complication.

\section{Conclusion}

This study aimed to identify common practice patterns among pediatric ophthalmologists on a difficult disease given the vulnerable patient population and the long-term burden of effects from the disease as well as the treatment modality. Despite previous study findings, questions about 
bevacizumab continue to persist, particularly systemic effects of anti-VEGF therapy and frequency and duration of follow-up. These clinical dilemmas may be answered by larger retrospective studies, as well as by prospective trials in clinical centers where pediatric ophthalmologists are already comfortable with bevacizumab therapy. In future studies, investigating recurrence rates, treatment of recurring cases, and surveying long-term systemic complications would be beneficial additions to the data analysis.

\section{Acknowledgment}

The study was conducted at Children's Hospital and Medical Center and the University of Nebraska Medical Center.

\section{Disclosure}

The authors report no conflicts of interest in this work.

\section{References}

1. Wallace DK, Wu KY. Current and future trends in treatment of severe retinopathy of prematurity. Clin Perinatol. 2013;40(2):297-310.

2. Good WV; Early Treatment for Retinopathy of Prematurity Cooperative Group. Final results of the Early Treatment for Retinopathy of Prematurity (ETROP) randomized trial. Trans Am Ophthalmol Soc. 2004; 102:233-248.

3. Yonekawa $\mathrm{Y}, \mathrm{Wu} \mathrm{WC}$, Nitulescu $\mathrm{CE}$, et al. Progressive retinal detachment in infants with retinopathy of prematurity treated with intravitreal bevacizumab or ranibizumab. Retina. Epub 2017 May 3.

4. Wu WC, Kuo HK, Yeh PT, Yang CM, Lai CC, Chen SN. An updated study of the use of bevacizumab in the treatment of patients with prethreshold retinopathy of prematurity in Taiwan. Am J Ophthalmol. 2013;155(1):150-158.

5. Mintz-Hittner HA, Kennedy KA, Chuang AZ; BEAT-ROP Cooperative Group. Efficacy of intravitreal bevacizumab for stage 3+ retinopathy of prematurity. N Engl J Med. 2011;364(7):603-615.

6. Hu J, Blair MP, Shapiro MJ, Lichtenstein SJ, Galasso JM, Kapur R. Reactivation of retinopathy of prematurity after bevacizumab injection. Arch Ophthalmol. 2012;130(8):1000-1006.

7. Wu WC, Yeh PT, Chen SN, Yang CM, Lai CC, Kuo HK. Effects and complications of bevacizumab use in patients with retinopathy of prematurity: a multicenter study in Taiwan. Ophthalmology. 2011; 118(1):176-183.

8. Nazari H, Modarres M, Parvaresh MM, Ghasemi Falavarjani K. Intravitreal bevacizumab in combination with laser therapy for the treatment of severe retinopathy of prematurity (ROP) associated with vitreous or retinal hemorrhage. Graefes Arch Clin Exp Ophthalmol. 2010; 248(12):1713-1718.

Clinical Ophthalmology

\section{Publish your work in this journal}

Clinical Ophthalmology is an international, peer-reviewed journal covering all subspecialties within ophthalmology. Key topics include: Optometry; Visual science; Pharmacology and drug therapy in eye diseases; Basic Sciences; Primary and Secondary eye care; Patient Safety and Quality of Care Improvements. This journal is indexed on
9. Dorta P, Kychenthal A. Treatment of type 1 retinopathy of prematurity with intravitreal bevacizumab (Avastin). Retina. 2010;30(Suppl 4): S24-S31.

10. Breier G, Albrecht U, Sterrer S, Risau W. Expression of vascular endothelial growth factor during embryonic angiogenesis and endothelial cell differentiation. Development. 1992;114(2):521-532.

11. Härd AL, Hellström A. On safety, pharmacokinetics and dosage of bevacizumab in ROP treatment - a review. Acta Paediatr. 2011; 100(12):1523-1527.

12. Law JC, Recchia FM, Morrison DG, Donahue SP, Estes RL. Intravitreal bevacizumab as adjunctive treatment for retinopathy of prematurity. J AAPOS. 2010;14(1):6-10.

13. Mintz-Hittner HA, Kuffel RR Jr. Intravitreal injection of bevacizumab (avastin) for treatment of stage 3 retinopathy of prematurity in zone I or posterior zone II. Retina. 2008;28(6):831-838.

14. Martínez-Castellanos MA, Schwartz S, Hernández-Rojas ML, et al. Long-term effect of antiangiogenic therapy for retinopathy of prematurity up to 5 years of follow-up. Retina. 2013;33(2):329-338.

15. Lien R, Yu MH, Hsu KH, et al. Neurodevelopmental outcomes in infants with retinopathy of prematurity and bevacizumab treatment. PLoS One. 2016;11(1):e0148019.

16. Wu WC, Lien R, Liao PJ, et al. Serum levels of vascular endothelial growth factor and related factors after intravitreous bevacizumab injection for retinopathy of prematurity. JAMA Ophthalmol. 2015; 133(4):391-397.

17. Patel CK, Fung THM, Muqit MMK, et al. Non-contact ultra-widefield imaging of retinopathy of prematurity using the Optos dual wavelength scanning laser ophthalmoscope. Eye. 2013;27(5):589-596.

18. Mintz-Hittner HA, Kennedy KA, Chuang AZ. Efficacy of intravitreal bevacizumab for stage 3+ retinopathy of prematurity. NEngl JMed.2011; 364(7):603-615.

19. Harder BC, von Baltz S, Jonas JB, Schlichtenbrede FC. Intravitreal lowdosage bevacizumab for retinopathy of prematurity. Acta Ophthalmol. 2014;92(6):577-581.

20. Wallace DK, Kraker RT, Freedman SF, et al. Assessment of lower doses of intravitreous bevacizumab for retinopathy of prematurity: a phase 1 dosing study. JAMA Ophthalmol. 2017;135(6):654-656.

21. Stahl A, Krohne TU, Eter N, et al. Comparing alternative ranibizumab dosages for safety and efficacy in retinopathy of prematurity: a randomized clinical trial. JAMA Pediatr. 2018;172(3):278-286.

22. Lee BJ, Kim JH, Heo H, Yu YS. Delayed onset atypical vitreoretinal traction band formation after an intravitreal injection of bevacizumab in stage 3 retinopathy of prematurity. Eye. 2012;26(7):903-910.

23. Lepore D, Quinn GE, Molle F, et al. Follow-up to age 4 years of treatment of type 1 retinopathy of prematurity intravitreal bevacizumab injection versus laser: fluorescein angiographic findings. Ophthalmology. 2018;125(2):218-226.

24. Harper CA 3rd, Wright LM, Young RC, Read SP, Chang EY. Fluorescein angiographic evaluation of peripheral retinal vasculature after primary intravitreal ranibizumab for retinopathy of prematurity. Retina. Epub 2018 January 3.
PubMed Central and CAS, and is the official journal of The Society of Clinical Ophthalmology (SCO). The manuscript management system is completely online and includes a very quick and fair peer-review system, which is all easy to use. Visit http://www.dovepress.com/ testimonials.php to read real quotes from published authors. 